\title{
An investigation on a new class of harmonic mappings
}

Emel Yavuz Duman ${ }^{1 *}$, Yaşar Polatoğlu' and Yasemin Kahramaner ${ }^{2}$

"Correspondence:

e.yavuz@iku.edu.tr

${ }^{1}$ Department of Mathematics and Computer Science, İstanbul Kültür

University, Ataköy Campus, Bakırköy, Istanbul, 34156, Turkey

Full list of author information is

available at the end of the article

\begin{abstract}
In the present paper, we give an extension of the idea which was introduced by Sakaguchi (J. Math. Soc. Jpn. 11:72-75, 1959), and we give some applications of this extended idea for the investigation of the class of harmonic mappings.
\end{abstract}

MSC: Primary 30C45; 30C55

Keywords: harmonic mappings; distortion theorem; grow theorem

\section{Introduction}

Let $\mathbb{D}=\{z|| z \mid<1\}$ be the open unit disc in the complex plane $\mathbb{C}$. A complex valued harmonic function $f: \mathbb{D} \rightarrow \mathbb{C}$ has the representation

$$
f=h(z)+\overline{g(z)}
$$

where $h(z)$ and $g(z)$ are analytic in $\mathbb{D}$ and have the following power series expansions:

$$
h(z)=\sum_{n=0}^{\infty} a_{n} z^{n}, \quad g(z)=\sum_{n=0}^{\infty} b_{n} z^{n}, \quad z \in \mathbb{D}
$$

where $a_{n}, b_{n} \in \mathbb{C}, n=0,1,2, \ldots$ Choose $g(0)=0$ (i.e., $b_{0}=0$ ), so the representation (1.1) is unique in $\mathbb{D}$ and is called the canonical representation of $f$ in $\mathbb{D}$. It is convenient to make further normalization (without loss of generality) $h(0)=0$ (i.e., $a_{0}=0$ ) and $h^{\prime}(0)=1$ (i.e., $a_{1}=1$ ). The family of such functions $f$ is denoted by $\mathcal{S}_{\mathcal{H}}$ [1]. It is known that if $f$ is a sensepreserving harmonic mapping of $\mathbb{D}$ onto some other region, then by Lewy's theorem its Jacobian is strictly positive, i.e.,

$$
J_{f(z)}=\left|h^{\prime}(z)\right|^{2}-\left|g^{\prime}(z)\right|^{2}>0
$$

Equivalently, the inequality $\left|g^{\prime}(z)\right|<\left|h^{\prime}(z)\right|$ holds for all $z \in \mathbb{D}$. The family of all functions $f \in$ $\mathcal{S}_{\mathcal{H}}$ with the additional property that $g^{\prime}(0)=0$ (i.e., $\left.b_{1}=0\right)$ is denoted by $\mathcal{S}_{\mathcal{H}}^{0}$ [1]. Observe that the classical family of univalent functions $\mathcal{S}$ consists of all functions $f \in \mathcal{S}_{\mathcal{H}}^{0}$ such that $g(z)=0$ for all $z \in \mathbb{D}$. Thus, it is clear that $\mathcal{S} \subset \mathcal{S}_{\mathcal{H}}^{0} \subset \mathcal{S}_{\mathcal{H}}$.

Let $\Omega$ be the family of functions $\phi(z)$ regular in the open unit disc $\mathbb{D}$ and satisfy the conditions $\phi(0)=0,|\phi(z)|<1$ for all $z \in \mathbb{D}$.

\section{Springer}

O2013 Yavuz Duman et al.; licensee Springer. This is an Open Access article distributed under the terms of the Creative Commons Attribution License (http://creativecommons.org/licenses/by/2.0), which permits unrestricted use, distribution, and reproduction in any medium, provided the original work is properly cited. 
Denote by $\mathcal{P}$ the family of functions $p(z)=1+p_{1} z+p_{2} z^{2}+\cdots$ regular in $\mathbb{D}$ such that $p(z)$ in $\mathcal{P}$ if and only if

$$
p(z)=\frac{1+\phi(z)}{1-\phi(z)}
$$

for some $\phi(z) \in \Omega$ and every $z \in \mathbb{D}$.

If $s(z)=z+c_{2} z^{2}+\cdots$ is regular in the open unit disc $\mathbb{D}$ and satisfies the condition

$$
\operatorname{Re}\left(e^{i \alpha} z \frac{s^{\prime}(z)}{s(z)}\right)>0, \quad z \in \mathbb{D}
$$

for some real $\alpha,|\alpha|<\pi / 2$, then $s(z)$ is said to be an $\alpha$-spirallike function in $\mathbb{D}[2,3]$. Such functions are known to be univalent [4]. The class of such functions is denoted by $\mathcal{S}_{\alpha}^{*}$.

Let $F_{1}(z)=z+\alpha_{2} z^{2}+\alpha_{3} z^{3}+\cdots$ and $F_{2}(z)=z+\beta_{2} z^{2}+\beta_{3} z^{3}+\cdots$ be analytic functions in $\mathbb{D}$. If there exists a function $\phi(z) \in \Omega$ such that $F_{1}(z)=F_{2}(\phi(z))$ for every $z \in \mathbb{D}$, then we say that $F_{1}(z)$ is subordinate to $F_{2}(z)$ and we write $F_{1} \prec F_{2}$. We also note that if $F_{1} \prec F_{2}$, then $F_{1}(\mathbb{D}) \subset F_{2}(\mathbb{D})[2,3]$. We also note that $w(z)=g^{\prime}(z) / h^{\prime}(z)$ is the analytic second dilatation of $f$ and $|w(z)|<1$ for every $z \in \mathbb{D}$.

In this paper we investigate the class of harmonic mappings defined by

$$
\mathcal{S}_{\mathcal{H}(\alpha)}=\left\{f=h(z)+\overline{g(z)} \mid w(z) \prec \frac{1+z}{1-z}, h(z) \in \mathcal{S}_{\alpha}^{*}\right\} .
$$

For this aim we need the following theorems and lemmas.

Theorem 1.1 [5] Let $s(z) \in \mathcal{S}_{\alpha}^{*}$, then

$$
r F(\cos \alpha,-r) \leq|s(z)| \leq r F(\cos \alpha, r)
$$

and

$$
\begin{aligned}
& {[(1-r) \cos \alpha-(1+r) \sin \alpha] F(\cos \alpha,-r)} \\
& \quad \leq\left|s^{\prime}(z)\right| \leq[(1+r) \cos \alpha+(1-r) \sin \alpha] F(\cos \alpha, r),
\end{aligned}
$$

where

$$
F(\cos \alpha, r)=\frac{1}{(1+r)^{\cos \alpha(\cos \alpha-1)}(1-r)^{\cos \alpha(\cos \alpha+1)}}
$$

for all $|z|=r<1$ and $|\alpha|<\pi / 2$.

Lemma 1.2 [6] Let $\phi(z)$ be regular in the unit disc $\mathbb{D}$ with $\phi(0)=0$, then if $|\phi(z)|$ attains its maximum value on the circle $|z|=r$ at the point $z_{1}$, one has $z_{1} \phi^{\prime}\left(z_{1}\right)=k \phi\left(z_{1}\right)$ for some $k \geq 1$.

Lemma 1.3 [7] If $s_{1}(z)$ and $s_{2}(z)$ are regular in $\mathbb{D}, s_{1}(0)=s_{2}(0), s_{2}(z)$ maps $\mathbb{D}$ onto a many-sheeted region which is starlike with respect to the origin, and $s_{1}^{\prime}(z) / s_{2}^{\prime}(z) \in \mathcal{P}$, then $s_{1}(z) / s_{2}(z) \in \mathcal{P}$. 
Lemma 1.4 [8] Let $f=h(z)+\overline{g(z)}$ be an element of $\mathcal{S}_{\mathcal{H P S T}(\alpha)^{*} \text {, then }}$

$$
\frac{\left|b_{1}\right|-r}{1-\left|b_{1}\right| r} \leq\left|\frac{g^{\prime}(z)}{h^{\prime}(z)}\right| \leq \frac{\left|b_{1}\right|+r}{1+\left|b_{1}\right| r}
$$

for all $|z|=r<1$. This inequality is sharp because the extremal function is

$$
e^{i \alpha} \frac{g^{\prime}(z)}{h^{\prime}(z)}=\frac{z+b}{1+\bar{b} z}
$$

where $b=e^{i \alpha} b_{1}$.

\section{Main results}

Theorem 2.1 $s(z) \in \mathcal{S}_{\alpha}^{*}$ if and only if

$$
z \frac{s^{\prime}(z)}{s(z)}-1 \prec \frac{2 \cos \alpha e^{-i \alpha} z}{1-z}
$$

for all $z \in \mathbb{D}$.

Proof Let $s(z)$ in $\mathcal{S}_{\alpha}^{*}$, then we have

$$
e^{i \alpha} z \frac{s^{\prime}(z)}{s(z)}=\cos \alpha \frac{1+\phi(z)}{1-\phi(z)}+i \sin \alpha
$$

or

$$
e^{i \alpha} z \frac{s^{\prime}(z)}{s(z)}=e^{i \alpha} \frac{1+\phi(z)}{1-\phi(z)}
$$

for some $\phi(z) \in \Omega$ and all $z \in \mathbb{D}$. Thus

$$
\begin{aligned}
z \frac{s^{\prime}(z)}{s(z)}-1 & =\frac{1+e^{-2 i \alpha} \phi(z)}{1-\phi(z)}-1 \\
& =\frac{1+(\cos 2 \alpha-i \sin 2 \alpha) \phi(z)-1+\phi(z)}{1-\phi(z)} \\
& =\frac{2 \cos \alpha e^{-i \alpha} \phi(z)}{1-\phi(z)}
\end{aligned}
$$

for some $\phi(z) \in \Omega$ and all $z \in \mathbb{D}$. Since $\phi(z) \in \Omega$, we have that (2.1) is true. The sufficient part of the proof can be seen by following the above steps in the opposite direction by considering the subordination principle.

Theorem 2.2 Let $f=h(z)+\overline{g(z)}$ be an element of $\mathcal{S}_{\mathcal{H}(\alpha)}$. If $w(z)=\frac{g^{\prime}(z)}{h^{\prime}(z)} \in \mathcal{P}$, then $\frac{g(z)}{h(z)} \in \mathcal{P}$ for all $z \in \mathbb{D}$.

Proof A version of this theorem was proved by Sakaguchi for a univalent starlike function [7, 9]. Since $f=h(z)+\overline{g(z)} \in \mathcal{S}_{\mathcal{H}(\alpha)}$, then $h(z)$ and $g(z)$ are regular in $\mathbb{D}$ and $h(0)=$ $g(0)=0$. On the other hand, we have

$$
w(z)=\frac{g^{\prime}(z)}{h^{\prime}(z)} \in \mathcal{P} \quad \text { if and only if } \quad \frac{g^{\prime}(z)}{h^{\prime}(z)} \prec \frac{1+z}{1+z}
$$


for all $z \in \mathbb{D}$. Geometrically, this means that $\frac{g^{\prime}(z)}{h^{\prime}(z)}$ maps $\mathbb{D}$ inside the open disc centered on the real axis with diameter end points $\frac{1-r}{1+r}$ and $\frac{1+r}{1-r}$. Now we define a function $\phi(z)$ by

$$
\frac{g(z)}{h(z)}=\frac{1+\phi(z)}{1-\phi(z)} \quad(z \in \mathbb{D})
$$

Then $\phi(z)$ is analytic in $\mathbb{D}$, and $\phi(0)=0$. On the other hand,

$$
w(z)=\frac{g^{\prime}(z)}{h^{\prime}(z)}=\frac{2 z \phi^{\prime}(z)}{1-\phi(z)} \frac{1}{e^{i \alpha}\left(1+e^{-2 i \alpha}\right) \phi(z)}+\frac{1+\phi(z)}{1-\phi(z)} \quad(z \in \mathbb{D}) .
$$

Now, it is easy to realize that the subordination (2.2) is equivalent to $|\phi(z)|<1$ in (2.3) for all $z \in \mathbb{D}$. Indeed, assume to the contrary that there exists $z_{1} \in \mathbb{D}$ such that $\left|\phi\left(z_{1}\right)\right|=1$. Then by Jack's lemma (Lemma 1.2), $z_{1} \phi^{\prime}\left(z_{1}\right)=k \phi\left(z_{1}\right), k \geq 1$, for such $z_{1}$ we have

$$
w\left(z_{1}\right)=\frac{g^{\prime}\left(z_{1}\right)}{h^{\prime}\left(z_{1}\right)}=\frac{2 k \phi\left(z_{1}\right)}{1-\phi\left(z_{1}\right)} \frac{1}{e^{i \alpha}\left(1+e^{-2 i \alpha}\right) \phi\left(z_{1}\right)}+\frac{1+\phi\left(z_{1}\right)}{1-\phi\left(z_{1}\right)}=w\left(\phi\left(z_{1}\right)\right) \notin w(\mathbb{D}),
$$

since $\left|\phi\left(z_{1}\right)\right|=1$ and $k \geq 1$. But this is a contradiction to the condition $w(z)=\frac{g^{\prime}(z)}{h^{\prime}(z)} \prec \frac{1+z}{1-z}$, and so the assumption is wrong, i.e., $|\phi(z)|<1$ for all $z \in \mathbb{D}$.

Remark 2.3 Theorem 2.2 is an extension of Lemma 1.3 to the harmonic mappings.

Corollary 2.4 Let $f=h(z)+\overline{g(z)}$ be an element of $\mathcal{S}_{\mathcal{H}(\alpha)}$, then

$$
\frac{r(1-r)}{1+r} F(\cos \alpha,-r) \leq|g(z)| \leq \frac{r(1+r)}{1-r} F(\cos \alpha, r)
$$

and

$$
\begin{aligned}
& {[(1-r) \cos \alpha-(1+r) \sin \alpha] \frac{1-r}{1+r} F(\cos \alpha,-r)} \\
& \quad \leq\left|g^{\prime}(z)\right| \leq[(1+r) \cos \alpha+(1-r) \sin \alpha] \frac{1+r}{1-r} F(\cos \alpha, r),
\end{aligned}
$$

where $F(\cos \alpha, r)$ is given by (1.6) for all $|z|=r<1$ and $|\alpha|<\pi / 2$.

Proof The proof of this theorem is a simple consequence of Theorem 1.1 and Theorem 2.2 since

$$
\operatorname{Re} w(z)=\operatorname{Re} \frac{g^{\prime}(z)}{h^{\prime}(z)} \quad \Rightarrow \quad \frac{g^{\prime}(z)}{h^{\prime}(z)} \prec \frac{1+z}{1-z} \quad \Rightarrow \quad \frac{1-r}{1+r} \leq\left|\frac{g^{\prime}(z)}{h^{\prime}(z)}\right| \leq \frac{1+r}{1-r},
$$

then

$$
\left|h^{\prime}(z)\right| \frac{1-r}{1+r} \leq\left|g^{\prime}(z)\right| \leq\left|h^{\prime}(z)\right| \frac{1+r}{1-r}
$$

and

$$
\operatorname{Re} \frac{g(z)}{h(z)}>0 \Rightarrow \frac{g(z)}{h(z)} \prec \frac{1+z}{1-z} \Rightarrow \frac{1-r}{1+r} \leq\left|\frac{g(z)}{h(z)}\right| \leq \frac{1+r}{1-r},
$$


then

$$
|h(z)| \frac{1-r}{1+r} \leq|g(z)| \leq|h(z)| \frac{1+r}{1-r} .
$$

Corollary 2.5 Let $f=h(z)+\overline{g(z)}$ be an element of $\mathcal{S}_{\mathcal{H}(\alpha)}$, then

$$
\begin{aligned}
& \frac{[(1-r) \cos \alpha-(1+r) \sin \alpha]^{2}(F(\cos \alpha,-r))^{2}\left(1-r^{2}\right)\left(1-\left|b_{1}\right|^{2}\right)}{\left(1+\left|b_{1}\right| r\right)^{2}} \\
& \quad \leq J_{f(z)} \leq \frac{[(1+r) \cos \alpha+(1-r) \sin \alpha]^{2}(F(\cos \alpha, r))^{2}\left(1-r^{2}\right)\left(1-\left|b_{1}\right|^{2}\right)}{\left(1-\left|b_{1}\right| r\right)^{2}}
\end{aligned}
$$

where $F(\cos \alpha, r)$ is given by (1.6) for all $|z|=r<1,|\alpha|<\pi / 2$ and $J_{f(z)}$ is the Jacobian off defined by $J_{f(z)}=\left|h^{\prime}(z)\right|^{2}-\left|g^{\prime}(z)\right|^{2}$ for all $z \in \mathbb{D}$.

Proof The proof of this corollary is a simple consequence of Lemma 1.1 and Lemma 1.4.

\section{Competing interests}

The authors declare that they have no competing interests.

\section{Authors' contributions}

All authors jointly worked on the results and they read and approved the final manuscript.

\section{Author details}

${ }^{1}$ Department of Mathematics and Computer Science, İstanbul Kültür University, Ataköy Campus, Bakırköy, İstanbul, 34156

Turkey. ${ }^{2}$ Department of Mathematics, İstanbul Commerce University, Üsküdar Campus, Üsküdar, İstanbul, 34672, Turkey.

Received: 28 December 2012 Accepted: 28 August 2013 Published: 07 Nov 2013

\section{References}

1. Duren, P: Harmonic Mappings in the Plane. Cambridge University Press, Cambridge (2004)

2. Goodman, AW: Univalent Functions, vol. I. Mariner Pub. Comp. Inc., Tampa (1983)

3. Goodman, AW: Univalent Functions, vol. II. Mariner Pub. Comp. Inc., Tampa (1983)

4. Špaček, L: Contribution à la théorie des fonctions univalentes. Čas. Pěst. Mat. 62, 12-19 (1932)

5. Polatoğlu, Y: Grow and distortion theorem for Janowski $\alpha$-spirallike functions in the unit disc. Stud. Univ. Babeş-Bolyai, Math. 57(2), 255-259 (2012)

6. Jack, IS: Functions starlike and convex of order $\alpha$. J. Lond. Math. Soc. 3, 469-474 (1971)

7. Libera, RJ: Some classes of regular univalent functions. Proc. Am. Math. Soc. 16, 755-758 (1965)

8. Aydoğan, M, Yavuz Duman, E, Polatoğlu, Y, Kahramaner, Y: Harmonic function for which the second dilatation is $\alpha$-spiral. J. Inequal. Appl. 2012, 262 (2012)

9. Sakaguchi, K: On a certain univalent mappings. J. Math. Soc. Jpn. 11, $72-75$ (1959)

10.1186/1029-242X-2013-478

Cite this article as: Yavuz Duman et al.: An investigation on a new class of harmonic mappings. Journal of Inequalities and Applications 2013, 2013:478 